\title{
NÍVEIS DE POTÊNCIA SONORA EMITIDOS POR TRATOR AGRÍCOLA EM CONDIÇÕES ESTÁTICAS E DINÂMICAS ${ }^{1}$
}

\author{
Aline Dantas da Silva Alves ${ }^{2}$, Fernanda Rodrigues Lima da Costa ${ }^{2}$, \\ Jorge Wilson Cortez ${ }^{2}$, Alan Christie da Silva Dantas ${ }^{3}$, Hideo de Jesus Nagahama ${ }^{2}$
}

\begin{abstract}
SOUND POWER LEVEL EMITTED BY AGRICULTURAL TRACTOR UNDER STATIC AND DINAMIC CONDITIONS
\end{abstract}

The noise produced by farm machinery may cause hearing problems to its operators along the time. The objective of this study was to evaluate the sound power level emitted by agricultural tractor under static and dynamic conditions, in relation to the IEC651 and IEC61672 norms. It was conducted at the Universidade Federal do Vale do São Francisco, in Petrolina, Pernambuco State, Brazil, in 2009, by using a Valtra 785 TDA tractor without cabin and equipment to measure its sound power level. The measurements were: static (anterior, posterior, right, and left positions, in relation to the operator's seat, for each meter along the maximum distance of $10 \mathrm{~m}$, and different engine rotation speeds, with two measuring devices) and dynamic (five speeds on concrete and soil test tracks, with two devices). Two measurement devices were used to evaluate the sound power level. For the devices in accordance with the IEC651 and IEC62672 norms, the second one always presented higher sound power levels, due to the fact that the first one was equipped with a wind protector. In all positions, the sound power level was higher than allowed by law. As the engine rotation speed increases, the sound power level also increases. It was noticed that both test tracks can be used for sound power level analysis, due to their similar results. The tractor speed affected its sound power level, with higher values for longer gears.

KEY-WORDS: Noise; ergonomics; agricultural mechanization.

\section{INTRODUÇÃO}

Até recentemente, o projeto dos tratores agrícolas centrava-se na maximização da eficiência do trator, em detrimento do fator humano. No entanto, devido ao aumento no rigor das normas de segurança de trabalho, há tendência para a melhoria das condições de ergonomia e segurança do operador, visando a melhorar as condições de trabalho, diminuir o nível

\section{RESUMO}

O ruído das máquinas agrícolas, com o passar do tempo, pode provocar problemas auditivos em seus operadores. O objetivo deste trabalho foi avaliar o nível de potência sonora emitido por trator agrícola, em condições estáticas e dinâmicas, comparando-o às normas IEC651 e IEC61672. O trabalho foi realizado na Universidade Federal do Vale do São Francisco, Petrolina (PE), em 2009, utilizando-se um trator Valtra, modelo 785 TDA sem cabine, e medidores do nível de potência sonora. As medições foram: estática (posições anterior, posterior, direita e esquerda do assento do operador a cada metro, até a distância máxima de $10 \mathrm{~m}$, e diferentes rotações do motor, com dois equipamentos de medição) e dinâmica (cinco velocidades de deslocamento, em pista de concreto e terra batida, utilizandose dois equipamentos de medição). Para os equipamentos que atendem às normas IEC651 e IEC61672, o segundo sempre apresentou maiores valores do nível de potência sonora, devido ao primeiro ser utilizado com protetor de vento. Em todas as condições, o nível de potência sonora foi maior do que o permitido pela legislação. À medida que se aumenta a rotação do motor, ocorre incremento no nível de potência sonora. Verificouse que as pistas de terra batida e concreto podem ser utilizadas para análise do nível de potência sonora, devido aos resultados similares apresentados. A velocidade de deslocamento do trator influenciou no nível de potência sonora, sendo que as marchas mais longas apresentaram maiores valores.

PALAVRAS-CHAVE: Ruído; ergonomia; mecanização agrícola.

de fadiga ao qual este está exposto, diminuir o risco de acidentes e aumentar a produtividade e qualidade do trabalho (Alves 2009).

O operador necessita não apenas de conforto, mas sentir-se em ambiente seguro, no seu posto de operação, sendo que o estresse pode afetar o desempenho e a eficiência do trabalho humano, aumentando a fadiga e o desconforto. Outro fator desfavorável, e que está em constante aumento de intensidade, é

1. Trabalho recebido em maio/2010 e aceito para publicação em jan./2011 (nº registro: PAT 9741/ DOI 10.5216/pat.v41i1.9741).

2. Universidade Federal do Vale do São Francisco, Petrolina, PE, Brasil. E-mails: alineunivasf@gmail.com, fernanda.costazoo@gmail.com, jorge.cortez@univasf.edu.br, hideo.nagahama@univasf.edu.br.

3. Universidade Federal do Vale do São Francisco, Colegiado de Engenharia Mecânica, Juazeiro, BA, Brasil. E-mail: alan.dantas@univasf.edu.br. 
o ruído provocado pelo meio ambiente (Vigna et al. 2007). As alterações ocasionadas pelos altos níveis de potência sonora não resultam em efeitos imediatos, mas acumulativos, e vão se estabelecendo com o tempo (Noronha et al. 2005).

Os equipamentos utilizados para medição do nível de ruído, que é a intensidade sonora, podem avaliar a densidade do fluxo de energia, expressa em $\mathrm{W} \mathrm{m}^{-2}$ ou j s ${ }^{-1} \mathrm{~m}^{-2}$; a pressão sonora, em $\mathrm{N} \mathrm{m}^{-2}$ ou Pascal (Pa); ou o nível de potência sonora, em Bel (B), sendo, na prática, utilizado o submútiplo decibel (dB) (Mialhe 1996). Os equipamentos que medem a potência sonora são conhecidos, popularmente, como decibelímetros e devem atender a normas internacionais de padronização, como as da International Electrotechnical Commission (IEC). A norma IEC60651, ou somente IEC651, é a mais antiga e utiliza padrões mais simples, enquanto a norma IEC61672 é mais recente e utiliza padrões mais rígidos, ou seja, apresenta mudanças para integração à informática e inserção dos conceitos de metrologia, devendo a calibração ser realizada por laboratório credenciado pelo Instituto Nacional de Metrologia, Padronização e Qualidade Industrial (Inmetro).

Estudando níveis de potência sonora, em função do raio de afastamento, em derriçadoras mecânicas e pulverizadores portáteis motorizados, Cunha \& Teodoro (2006) observaram que os níveis de potência sonora diminuíam de intensidade, à medida que se afastavam das máquinas avaliadas, porém, encontraram valores superiores aos recomendados, em um raio de $10 \mathrm{~m}$ de distância, causando desconforto.

Baesso et al. (2008) concluíram que o conjunto trator-pulverizador apresentou níveis de potência sonora, próximo ao ouvido do operador, acima dos limites permitidos (85 $\mathrm{dB}(\mathrm{A}))$ pela Norma Regulamentadora (NR-15) do Ministério do Trabalho e Emprego. Os operadores deste conjunto mecanizado estão sujeitos a risco de hipoacusia, quando trabalham com a assistência de ar ligada.

Cortez et al. (2008) observaram que o aumento da rotação do motor do trator acarreta aumento no nível de potência sonora, mas, somente em rotações extremas $(2.200 \mathrm{rpm})$, o operador estaria sujeito à condição de estresse, podendo trabalhar apenas 6 horas por dia sem o uso de protetor auricular.

Tosin et al. (2009) avaliaram a potência sonora de dois tratores agrícolas $(55,2 \mathrm{~kW}$ e $80,9 \mathrm{~kW})$, em função das pistas (asfalto, concreto e solo firme), pressões dos pneus $(103,4 \mathrm{kPa}, 137,9 \mathrm{kPa}, 172,4 \mathrm{kPa}$ e $206,8 \mathrm{kPa})$ e velocidades $\left(3,60 \mathrm{~km} \mathrm{~h}^{-1}, 5,04 \mathrm{~km} \mathrm{~h}^{-1}\right.$ e $6,84 \mathrm{~km} \mathrm{~h}^{-1}$ ), e concluíram que a potência sonora não foi influenciada por nenhum destes fatores, mas pela potência do motor e a presença ou não de cabine.

Souza et al. (2004), ao avaliarem o nível de potência sonora no conjunto trator + recolhedora-trilhadora de feijão, nas velocidades $2,0 \mathrm{~km}^{-1} ; 2,5 \mathrm{~km}^{-1}$; e $3,0 \mathrm{~km}^{-1}$, concluíram que, ao se aumentar a velocidade, ocorreu diminuição no nível de potência sonora.

De acordo com o exposto, objetivou-se avaliar o nível de potência sonora emitido por um trator agrícola sem cabine, em condições estática (em função da rotação do motor e das posições) e dinâmica (pistas x velocidade de deslocamento), comparando-se as medições com decibelímetros, segundo as normas IEC651 e IEC61672.

\section{MATERIAL E MÉTODOS}

Os ensaios foram conduzidos no Campus de Ciências Agrárias da Universidade Federal do Vale do São Francisco (Univasf), em Petrolina (PE) (09¹9'26"S, 40³3'36"W e altitude de 393 m), em 09/09/2009. Segundo a classificação de Köppen (Brasil 1973), o clima da região apresenta-se como tropical semiárido, tipo BshW, caracterizado pela escassez e irregularidade de precipitações, com chuvas no verão e forte evaporação, em consequência das altas temperaturas.

Como fonte de potência, foi utilizado um trator da marca Valtra, modelo 785 TDA (tração dianteira auxiliar), com 55,2 kW (75 cv) no motor, fabricado em 2004, com 2.920 horas de uso e rotação de trabalho no motor a $2.040 \mathrm{rpm}$, que condiciona $540 \mathrm{rpm}$ na tomada de potência.

Os níveis de potência sonora foram determinados por meio de decibelímetros digitais, que apresentavam calibração de fábrica, por se tratarem de equipamentos novos: um da marca Minipa - modelo MSL-1325, com três faixas de medição (32-80 dB(A), 50-100 dB(A) e 80-130 dB(A)) e protetor de vento, atendendo à norma IEC651, tipo 2 (classes de 0 a 3), que o define para uso de campo; e outro da marca Homis - modelo 913 (H004-353), com três faixas de medição (30-70 dB(A), 60-100 dB(A) e 90-130 dB(A)), com Padrão IEC61672, tipo 1 (classes 1 e 2), sem protetor de vento.

Os equipamentos foram descritos pela norma que atende à Minipa - IEC651 e Homis - IEC61672. Ambos foram utilizados conforme MTE (2008), nos 
circuitos de resposta lenta (SLOW) e de equalização "A", sendo expressos em dB(A).

Em condição estática, para a avaliação do nível de potência sonora, em função da rotação do motor e dos equipamentos de medição, utilizou-se delineamento inteiramente casualizado (DIC), em esquema fatorial 2 x 15 (equipamentos x rotações no motor), com 3 repetições. Os equipamentos avaliados foram 0 IEC651 e IEC61672 e as rotações de 800-2.500 rpm, intercaladas a cada $100 \mathrm{rpm}$. Também, em condição estática, para a avaliação dos equipamentos, em função das posições (frente, traseira, direita e esquerda) e do raio de afastamento (distâncias em relação ao trator de 1-10 m), utilizou-se o delineamento inteiramente casualizado, em esquema fatorial $2 \times 4 \times 10$ (equipamentos x posições x distâncias em relação ao trator), com 3 repetições.

Em condição dinâmica, para avaliação do nível de potência sonora, em função das pistas, equipamentos e velocidades de deslocamento, utilizou-se o delineamento inteiramente casualizado, em esquema fatorial $2 \times 2 \times 5$ (pistas $\mathrm{x}$ equipamentos $\mathrm{x}$ velocidades), sendo duas pistas (concreto e terra batida), dois equipamentos (IEC 651 e IEC 61672) e cinco velocidades de deslocamento $\left(3,0 \mathrm{~km} \mathrm{~h}^{-1} ; 5,0 \mathrm{~km} \mathrm{~h}^{-1} ; 8,7 \mathrm{~km} \mathrm{~h}^{-1}\right.$; $10,7 \mathrm{~km} \mathrm{~h}^{-1}$; e 13,0 $\left.\mathrm{km} \mathrm{h}^{-1}\right)$.

As avaliações basearam-se no método descrito na NBR-9999 (ABNT 1987). Segundo esta norma, na posição e momento do ensaio de medição do nível de potência sonora, a temperatura ambiente deve estar entre $-5^{\circ} \mathrm{C}$ e $30^{\circ} \mathrm{C}$ e a velocidade do vento deve ser inferior a $5,0 \mathrm{~m} \mathrm{~s}^{-1}$, dados observados por meio de uma estação meteorológica instalada no Campus de Ciências Agrárias. No momento do experimento, a velocidade do vento e a temperatura foram de $26,0^{\circ} \mathrm{C}$ e $4,9 \mathrm{~m} \mathrm{~s}^{-1}$, respectivamente, atendendo às solicitações da norma. Como a velocidade do vento estava próxima à limite, para o aparelho que atende à norma IEC651, utilizou-se o protetor de vento, por se tratar de um equipamento de uso a campo.

Para realizar as medições, colocou-se o medidor de nível de potência sonora próximo ao ouvido do operador, $79 \mathrm{~cm}$ acima, $15 \mathrm{~cm}$ à frente e $20 \mathrm{~cm}$ lateralmente, em relação ao ponto de referência do assento (Seat Index Point - SIP), de acordo com a norma NBR 5353 (ABNT 1999). Ou seja, o microfone foi instalado ao lado da cabeça do operador que foi submetido ao nível de potência sonora (Mialhe 1996).

Para as avaliações na condição estática, com a variação da rotação do motor do trator de
800-2.500 rpm, medida pelo tacômetro, verificou-se o nível de potência sonora para cada intervalo de $100 \mathrm{rpm}$, para cada aparelho. Assim, as determinações foram realizadas a cada $5 \mathrm{~s}$, após um minuto de espera, para que o trator se estabilizasse na rotação pretendida. Para avaliação da potência sonora, em função dos raios de afastamento, as leituras foram tomadas em cada posição de 1-10 m, a intervalos de $1 \mathrm{~m}$, direcionadas para os lados direito e esquerdo e às partes anterior e posterior do trator, com os dois equipamentos (IEC651 e IEC61672). Em cada ponto, foram realizadas três leituras para cada condição, com o motor a $2.040 \mathrm{rpm}$, estado em que fornece $540 \mathrm{rpm}$ na tomada de potência.

Para a avaliação dinâmica, foram tomadas três medidas do nível de potência sonora, no percurso de $30 \mathrm{~m}$, com o trator em movimento, às velocidades teóricas de $3 \mathrm{~km} \mathrm{~h}^{-1}$ (L1), $5 \mathrm{~km} \mathrm{~h}^{-1}$ (L2), $8 \mathrm{~km} \mathrm{~h}^{-1}$ (H1), 10,7 $\mathrm{km} \mathrm{h}^{-1}$ (L3) e $13 \mathrm{~km} \mathrm{~h}^{-1}$ (H2) (adaptado de Mialhe 1996), com o motor a $2.040 \mathrm{rpm}$ e os dois aparelhos (IEC 651 e IEC 61672), nas duas condições de pista (concreto e terra batida), sendo as medidas realizadas a cada $5 \mathrm{~s}$.

Os dados obtidos em todos os ensaios foram analisados com o auxílio do software ESTAT(Unesp 1991). As médias do fator quantitativo, quando significativo a $5 \%$ (Teste $\mathrm{F}$ ), foram avaliadas por regressão polinomial e as médias dos fatores qualitativos, quando significativos a 5\% (Teste F), foram comparadas entre si pelo teste Tukey, a 5\%.

\section{RESULTADOS E DISCUSSÃO}

\section{Condição estática: rotação do motor x equipamentos de medição}

Para o nível de potência sonora (Tabela 1), houve influência significativa $(\mathrm{p} \leq 0,05)$ para os equipamentos $(\mathrm{E})$, rotação do motor $(\mathrm{R})$ e interação E x R. Observou-se que o nível de potência sonora (Tabela 1) foi maior para o equipamento que atende pela norma IEC61672. Este fato pode ser devido ao equipamento não possuir protetor de vento, enquanto o equipamento que atende pela IEC651 dele dispõe. Martinelli (2008) verificou que o aumento de $5 \mathrm{~dB}(\mathrm{~A})$ duplica a potência sonora, assim, pequenos acréscimos no nível de ruído (em $\mathrm{dB}$ ) acarretam grande aumento de potência sonora.

Quanto aos níveis de potência sonora encontrados nos dois equipamentos testados, ocorreu situação 
Tabela 1. Médias do nível de potência sonora $(\mathrm{dB}(\mathrm{A}))$ para os equipamentos utilizados (IEC651 e IEC61672) (Petrolina, PE, 2009).

\begin{tabular}{ccc}
\hline rpm & IEC651 & IEC61672 \\
\hline 800 & $81,00 \mathrm{~B}$ & $82,80 \mathrm{~A}$ \\
900 & $81,70 \mathrm{~B}$ & $86,10 \mathrm{~A}$ \\
1.000 & $82,90 \mathrm{~B}$ & $84,80 \mathrm{~A}$ \\
1.100 & $83,80 \mathrm{~B}$ & $85,70 \mathrm{~A}$ \\
1.200 & $85,00 \mathrm{~B}$ & $87,30 \mathrm{~A}$ \\
1.300 & $86,00 \mathrm{~B}$ & $87,70 \mathrm{~A}$ \\
1.400 & $87,20 \mathrm{~B}$ & $88,90 \mathrm{~A}$ \\
1.500 & $87,80 \mathrm{~B}$ & $89,70 \mathrm{~A}$ \\
1.600 & $88,70 \mathrm{~B}$ & $90,90 \mathrm{~A}$ \\
1.700 & $89,10 \mathrm{~B}$ & $91,40 \mathrm{~A}$ \\
1.800 & $90,10 \mathrm{~B}$ & $91,80 \mathrm{~A}$ \\
1.900 & $90,10 \mathrm{~B}$ & $92,00 \mathrm{~A}$ \\
2.000 & $91,20 \mathrm{~B}$ & $93,00 \mathrm{~A}$ \\
2.100 & $92,30 \mathrm{~B}$ & $93,80 \mathrm{~A}$ \\
2.200 & $92,70 \mathrm{~B}$ & $94,60 \mathrm{~A}$ \\
2.300 & $94,40 \mathrm{~B}$ & $96,40 \mathrm{~A}$ \\
2.400 & $94,80 \mathrm{~B}$ & $96,80 \mathrm{~A}$ \\
2.500 & $95,00 \mathrm{~B}$ & $96,90 \mathrm{~A}$ \\
\hline
\end{tabular}

Médias seguidas de mesma letra, na linha, não diferem entre si, pelo teste Tukey, a $5 \%$.

de desconforto para o operador a partir de $1.200 \mathrm{rpm}$, com relação à norma NR-15 (MTE 2008), pois está acima de $85 \mathrm{~dB}(\mathrm{~A})$, podendo gerar fadiga, desconcentração no serviço e diminuição na produtividade do trabalho (Fernandes 1990, Fernandes et al. 1991).

Com relação à análise de regressão, para os equipamentos utilizados no ensaio (Figura 1), observa-se que, à medida que aumenta a rotação do motor, ocorre incremento linear do nível de potência sonora emitido, para os dois equipamentos. Em condições de baixas rotações (1.100-1.200 rpm), observa-se que o

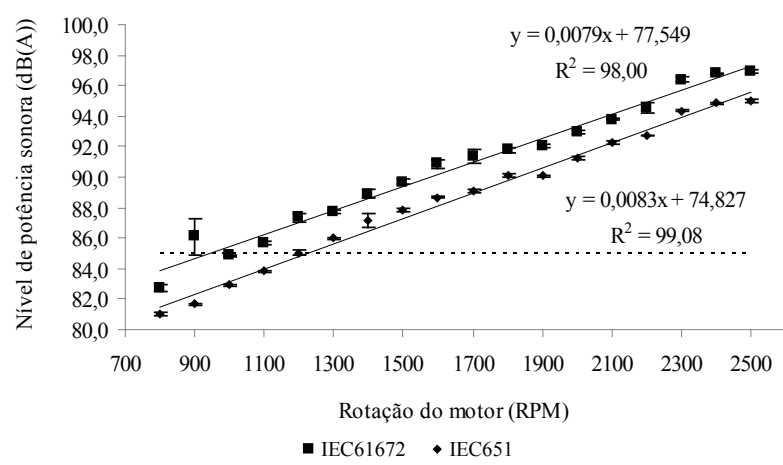

Figura 1. Nível de potência sonora para os equipamentos utilizados, em função da rotação do motor. A linha tracejada indica o limite máximo de potência sonora em que se pode trabalhar sem o uso de proteção auricular (Petrolina, PE, 2009). valor do nível de potência sonora extrapola os limites da NR-15. Deste modo, para qualquer operação com o trator, deve-se utilizar protetor auricular.

Cortez et al. (2008) verificaram que, para um trator de 55,2 kW (mesma potência deste trabalho), acima de $900 \mathrm{rpm}$, o nível de potência sonora observado foi significativo a $85 \mathrm{~dB}(\mathrm{~A})$. Caso o protetor auricular não seja utilizado, pode ocorrer risco de reações físicas, como aumento da pressão sanguínea e do ritmo cardíaco, com nível de potência sonora entre $65 \mathrm{~dB}(\mathrm{~A})$ e $85 \mathrm{~dB}(\mathrm{~A})$, e, entre $85 \mathrm{~dB}(\mathrm{~A})$ e $120 \mathrm{~dB}(\mathrm{~A})$, podem ocorrer alterações reversíveis ou irreversíveis no ouvido e perdas crescentes de percepção (Robin 1987 apud Mialhe 1996).

A diferença nas medições entre os equipamentos pode ser atribuída ao uso do protetor de vento, uma vez que os equipamentos apresentavam calibração de fábrica e eram novos.

\section{Condição estática: equipamentos $x$ posições $x$ raio de afastamento do trator}

Na síntese de análise de variância do nível de potência sonora para equipamentos, posições e raio de afastamento do trator, verificou-se significância $(\mathrm{p} \leq 0,05)$ para os fatores isoladamente e na interação posição $\mathrm{x}$ raio de afastamento.

O equipamento IEC61672 apresentou maior nível de potência sonora do que o IEC651, devido ao uso do protetor de vento, pelo segundo (Tabela 2). A posição do trator que apresentou, na média, o maior nível de potência sonora foi à frente e à direita, provavelmente devido à presença do motor e do alternador, respectivamente. Já a medida na posição à esquerda foi menor, mesmo com a presença do escapamento, por estar afastado do motor e a TDP (tomada de potência) estar desligada. Na média geral, nenhuma das

Tabela 2. Médias do nível de potência sonora, em função dos equipamentos utilizados (IEC651 e IEC61672) e da posição em relação ao trator (Petrolina, PE, 2009).

\begin{tabular}{lc}
\hline Aparelho & Potência sonora $(\mathrm{dB}(\mathrm{A}))$ \\
\hline IEC651 & $81,00 \mathrm{~B}$ \\
IEC61672 & $83,30 \mathrm{~A}$ \\
\hline Posição & Potência sonora $(\mathrm{dB}(\mathrm{A}))$ \\
\hline Frente & $83,40 \mathrm{~A}$ \\
Traseira & $79,20 \mathrm{C}$ \\
Direita & $83,20 \mathrm{~A}$ \\
Esquerda & $82,70 \mathrm{~B}$ \\
\hline
\end{tabular}

Médias seguidas de mesma letra, na coluna, não diferem entre si, pelo teste Tukey $(5 \%)$, para posição e aparelho. 
posições extrapolou o limite de $85 \mathrm{~dB}(\mathrm{~A})$, conforme a NR-15 (MTE 2008).

Pelo resultado da interação posição $\mathrm{x}$ raio de afastamento do trator (Tabela 3), observa-se que, para a posição frente, esquerda e direita, de maneira geral, as pessoas que trabalham a até quatro metros do trator devem utilizar o equipamento de proteção individual (EPI), e, para a parte traseira e sem a TDP ligada, a até um metro, pois os valores, até estas distâncias, extrapolam o limite da NR-15 de $85 \mathrm{~dB}(\mathrm{~A})$, para 8 horas de trabalho diário (MTE 2008). A parte traseira apresentou o menor valor de potência sonora, em todas as distâncias, enquanto as demais diferiram entre si, mas acima de seis metros, e dois e três metros, a parte traseira do trator apresentou o menor nível de potência sonora, sendo diferente das demais (frente, direita e esquerda). A parte esquerda do trator foi a que apresentou o maior valor de potência sonora a um metro, mas não diferiu da frente. Este fato pode estar ligado à presença do escapamento, que é fonte de ruído. Baesso et al. (2008) verificaram, com um trator de $73,6 \mathrm{~kW}(100 \mathrm{cv})$ acoplado ao pulverizador, que, até a distância de quatro metros, as pessoas que trabalhavam próximas deveriam utilizar EPI.

Observa-se que, próximo à origem da emissão, os níveis de potência sonora estão acima dos toleráveis $(85 \mathrm{~dB}(\mathrm{~A}))$, para 8 horas de exposição diária (MTE 2008). Souza et al. (2004) observaram que níveis de potência sonora acima de $75 \mathrm{~dB}(\mathrm{~A})$ podem causar desconforto acústico, prejuízo na comunicação, irritação e diminuição da produtividade de trabalho.

Tabela 3. Médias do nível de potência sonora $(\mathrm{dB}(\mathrm{A}))$, em função da posição e do raio de afastamento, em relação ao trator (Petrolina, PE, 2009).

\begin{tabular}{ccrcc}
\hline Distância $(\mathrm{m})$ & Frente & Traseira & Direita & Esquerda \\
\hline 1 & $93,80 \mathrm{AB}$ & $87,40 \mathrm{C}$ & $92,90 \mathrm{~B}$ & $94,50 \mathrm{~A}$ \\
2 & $88,80 \mathrm{~A}$ & $83,80 \mathrm{~B}$ & $89,40 \mathrm{~A}$ & $89,70 \mathrm{~A}$ \\
3 & $86,30 \mathrm{~A}$ & $81,60 \mathrm{~B}$ & $86,40 \mathrm{~A}$ & $85,90 \mathrm{~A}$ \\
4 & $85,00 \mathrm{~A}$ & $79,90 \mathrm{C}$ & $84,50 \mathrm{AB}$ & $83,40 \mathrm{C}$ \\
5 & $83,50 \mathrm{~A}$ & $78,10 \mathrm{C}$ & $83,80 \mathrm{~A}$ & $81,60 \mathrm{~B}$ \\
6 & $81,50 \mathrm{~A}$ & $78,90 \mathrm{~B}$ & $81,40 \mathrm{~A}$ & $80,30 \mathrm{~A}$ \\
7 & $80,40 \mathrm{~A}$ & $77,00 \mathrm{~B}$ & $80,30 \mathrm{~A}$ & $79,80 \mathrm{~A}$ \\
8 & $79,20 \mathrm{~A}$ & $76,40 \mathrm{~B}$ & $79,00 \mathrm{~A}$ & $78,10 \mathrm{~A}$ \\
9 & $78,40 \mathrm{~A}$ & $75,50 \mathrm{~B}$ & $77,50 \mathrm{~A}$ & $77,50 \mathrm{~A}$ \\
10 & $77,20 \mathrm{~A}$ & $73,80 \mathrm{~B}$ & $76,70 \mathrm{~A}$ & $76,70 \mathrm{~A}$ \\
\hline
\end{tabular}

Médias seguidas de mesma letra, na linha, não diferem entre si, pelo teste Tukey, a $5 \%$.
Para os demais raios de afastamento, os níveis de potência sonora foram diminuindo, concordando com observações realizadas por Cunha \& Teodoro (2006) e Silveira et al. (2007). O trator parado, com o motor em funcionamento a $2.040 \mathrm{rpm}$, apresentou maior valor $(94,50 \mathrm{~dB}(\mathrm{~A}))$ do lado esquerdo do operador, onde se localiza o escapamento.

Na síntese da análise de variância para regressão polinomial das distâncias, em relação às posições para o equipamento IEC651, todos os graus foram significativos e adotou-se o de maior valor $\left(3^{\circ}\right)$ para todas as posições de estudo, em virtude, também, de apresentar o maior $\mathrm{R}^{2}$.

Observa-se que a parte traseira do trator apresentou os menores valores do nível de potência sonora (Figura 2), enquanto as curvas da parte dianteira, esquerda e direita se mantiveram muito próximas. Baesso et. al. (2008) analisaram o nível de potência sonora a até $10 \mathrm{~m}$ do trator e utilizaram equação linear para sua representação, verificando, também, menor nível de potência sonora na parte traseira. Para as partes dianteira, direita e esquerda, as pessoas que se encontrarem a até $3 \mathrm{~m}$ devem utilizar algum tipo de protetor auricular. Na parte traseira, apenas a um metro de distância seria necessária a utilização de equipamentos de proteção auricular, para exposição de 8 horas diária, segundo a NR-15 (MTE 2008).

Na síntese da análise de variância para regressão polinomial do raio de afastamento do trator, em relação às posições para o equipamento IEC61672, as posições



Figura 2. Nível de potência sonora, em função das distâncias e posições do aparelho IEC651. A linha tracejada indica limite máximo de exposição diária $(85 \mathrm{~dB}(\mathrm{~A}))$, sem o uso de protetor auricular (Petrolina, PE, 2009). 
frente, traseira e esquerda foram significativas, em todos os níveis de regressão, e adotou-se a equação de maior grau $\left(3^{\circ}\right)$, pois a mesma apresentou o maior valor de $\mathrm{R}^{2}$ para a construção do gráfico de regressão. Para o lado direito do trator, foi significativa a regressão de segundo grau, com o maior valor de $\mathrm{R}^{2}$, sendo utilizada para a construção do gráfico de regressão.

Observa-se que a parte traseira do trator apresentou os menores valores do nível de potência sonora (Figura 3), mas é possível notar que, a partir de seis metros, há maior proximidade com as curvas das demais posições, enquanto as curvas da parte dianteira, esquerda e direita se mantiveram muito próximas. Para a parte dianteira, direita e esquerda do trator, pode-se verificar que as pessoas que trabalham a até quatro metros de distância do trator devem utilizar protetor auricular e, para a parte traseira, a até dois metros, deve-se utilizar o protetor, considerando-se, para ambos os casos, exposição máxima de 8 horas diárias, segundo a NR-15 (MTE 2008).

A partir dos resultados encontrados, sugere-se que o uso de EPI deve ser constante, ao se trabalhar com máquinas agrícolas, em virtude dos danos que podem provocar à saúde do operador. $\mathrm{O}$ uso de decibelímetros, seja o IEC651 ou o IEC61672, pode ajudar a determinar os níveis de ruído (no caso a potência sonora) que geram insalubridade ao operador, pois as diferenças apresentadas podem estar relacionadas ao uso do protetor de vento.

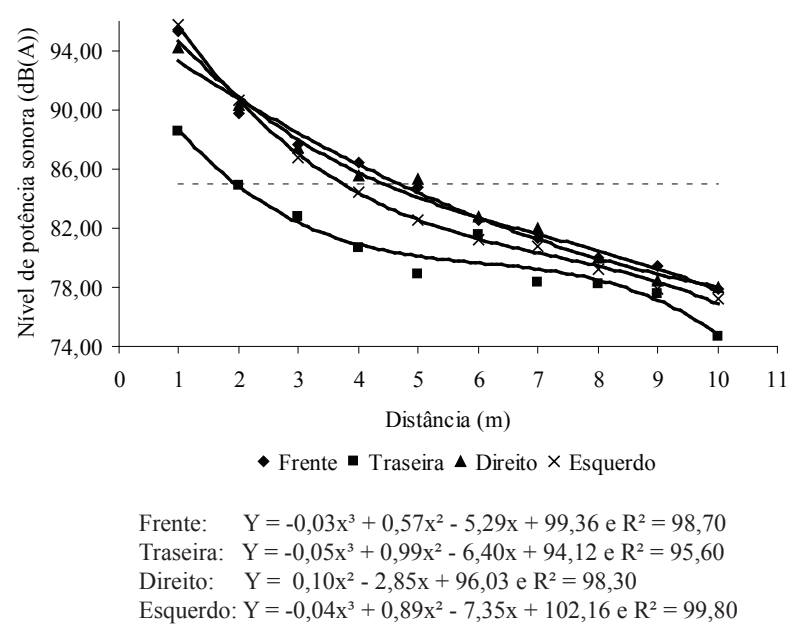

Figura 3. Nível de potência sonora para posição, em relação à distância do aparelho IEC61672. A linha tracejada indica limite máximo de exposição diária $(85 \mathrm{~dB}(\mathrm{~A}))$, sem o uso de protetor auricular (Petrolina, PE, 2009).
Condição dinâmica: pistas $x$ equipamentos $x$ velocidades

Na síntese da análise de variância para pista $\mathrm{x}$ equipamento $\mathrm{x}$ velocidade, verificou-se que não há diferença $(p>0,05)$ entre as pistas, para o nível de potência sonora, sendo o valor médio para pista de terra batida de $88,9 \mathrm{~dB}(\mathrm{~A})$ e de $89,2 \mathrm{~dB}(\mathrm{~A})$ para concreto, mas, em ambas as pistas, os valores estão acima do limite de $85 \mathrm{~dB}(\mathrm{~A})$, para uma jornada de 8 horas, segundo a NR-15 (MTE 2008). No entanto, ocorreu significância $(p \leq 0,05)$ para equipamentos e velocidades, indicando existir diferenças dentro dos fatores. Não se observou diferença $(p>0,05)$ para a interação pistas $\mathrm{x}$ equipamentos, indicando que os fatores agem independentemente. As interações pistas $(\mathrm{P}) \mathrm{x}$ velocidades (V) são apresentadas na Tabela 4 e equipamentos (E) $\mathrm{x}$ velocidades $(\mathrm{V})$ na Tabela 5. P x E x V foram significativas $(p \leq 0,05)$, indicando a forte influência da velocidade no nível de potência sonora.

Verifica-se que os maiores níveis de potência sonora ocorreram nas duas pistas de ensaio na maior velocidade (Tabela 4), exceto na pista de terra batida, na qual a velocidade de $5 \mathrm{~km} \mathrm{~h}^{-1}$ apresentou, também, o maior valor, diferenciando-se da pista de concreto. Analisando-se cada velocidade dentro das pistas, verifica-se que, à velocidade de $3,0 \mathrm{~km} \mathrm{~h}^{-1} \mathrm{e}$ $8,7 \mathrm{~km} \mathrm{~h}^{-1}$, não ocorreu diferença. No entanto, para

Tabela 4. Nível de potência sonora, em função das pistas $\mathrm{x}$ velocidades de deslocamento (Petrolina, PE, 2009).

\begin{tabular}{ccc}
\hline Velocidades & Pista de terra batida & Pista de concreto \\
\hline 3,0 & $87,62 \mathrm{Ba}$ & $87,52 \mathrm{Ca}$ \\
5,0 & $90,58 \mathrm{Aa}$ & $89,22 \mathrm{Bb}$ \\
8,7 & $87,97 \mathrm{Ba}$ & $88,70 \mathrm{BCa}$ \\
10,7 & $88,45 \mathrm{Bb}$ & $89,63 \mathrm{Ba}$ \\
13,0 & $89,83 \mathrm{Ab}$ & $91,25 \mathrm{Aa}$ \\
\hline
\end{tabular}

Médias seguidas de letras maiúsculas iguais, na coluna, e minúsculas, na linha, não diferem pelo teste Tukey, a 5\%.

Tabela 5. Nível de potência sonora, em função dos equipamentos de avaliação x velocidades de deslocamento (Petrolina, PE, 2009).

\begin{tabular}{cll}
\hline Velocidades & IEC651 & IEC61672 \\
\hline 3,0 & $81,25 \mathrm{Cb}$ & $93,88 \mathrm{Ca}$ \\
5,0 & $82,75 \mathrm{ABb}$ & $97,05 \mathrm{ABa}$ \\
8,7 & $82,90 \mathrm{ABb}$ & $93,77 \mathrm{Ca}$ \\
10,7 & $81,95 \mathrm{BCb}$ & $96,13 \mathrm{Ba}$ \\
13,0 & $83,45 \mathrm{Ab}$ & $97,63 \mathrm{Aa}$ \\
\hline
\end{tabular}

Médias seguidas de letras maiúsculas iguais, na coluna, e minúsculas, na linha, não diferem pelo teste Tukey, a 5\%. 
as velocidades de $10,7 \mathrm{~km} \mathrm{~h}^{-1} \mathrm{e} 13,0 \mathrm{~km} \mathrm{~h}^{-1}$, a pista de concreto apresentou o maior valor, provavelmente devido ao ruído provocado pelo contato pneu-concreto. Considerando-se os níveis de potência sonora permitidos pela NR-15 (MTE 2008), durante uma jornada de 8 horas, em ambas as pistas, os valores ficaram acima de $85 \mathrm{~dB}(\mathrm{~A})$, indicando a necessidade do uso de protetor auricular.

$\mathrm{Na}$ interação velocidade $\mathrm{x}$ equipamentos, verifica-se que ocorreu diferença entre os equipamentos, em todas as velocidades (Tabela 5), sendo que o aparelho que atende à norma IEC61672 apresentou maiores valores, acima do permitido pela NR-15 (MTE 2008), o que pode estar associado à falta do protetor de vento, enquanto os valores anotados pelo equipamento que atende à norma IEC651 apresentaram-se dentro do permitido, para uma jornada de trabalho de 8 horas. A velocidade de $13,0 \mathrm{~km} \mathrm{~h}^{-1}$ apresentou o maior nível de potência sonora, juntamente com a velocidade de $5,0 \mathrm{~km} \mathrm{~h}^{-1}$ e $8,7 \mathrm{~km} \mathrm{~h}^{-1}$, para o IEC651, e de $5,0 \mathrm{~km} \mathrm{~h}^{-1}$, para o IEC61672.

O fator velocidade foi significativo para os dois equipamentos (IEC651 e IEC61672). A regressão de primeiro grau foi significativa em ambos os equipamentos, no entanto, a escolha do modelo deve ocorrer com base no maior grau de significância, sendo, neste caso, o de terceiro grau, para ambos os equipamentos (Figura 4), ou seja, o grau de maior significância e que, correspondentemente, apresenta o maior $\mathrm{R}^{2}$. $\mathrm{O}$ $\mathrm{R}^{2}$ explica o quanto o modelo matemático se adequou aos dados, e, no caso da equação de primeiro grau, verificou-se $\mathrm{R}^{2}$ muito baixo, indicando a baixa representatividade do modelo.

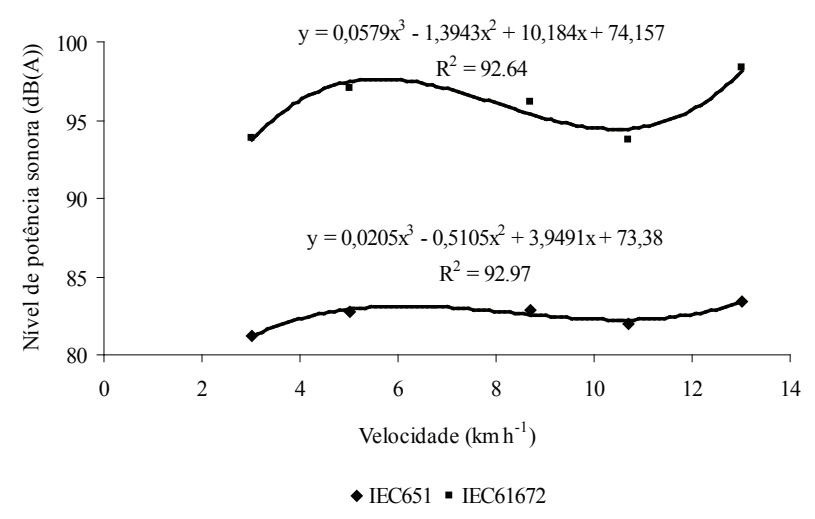

Figura 4. Nível de potência sonora, em função da velocidade de deslocamento x equipamentos de avaliação (Petrolina, PE, 2009).
O equipamento que atende à norma IEC61672 (Figura 4) apresentou os maiores valores de potência sonora, em todas as velocidades de deslocamento, acima do encontrado na NR-15 (MTE 2008). Considera-se que há diminuição na potência sonora no equipamento que atende à norma IEC651, devido ao uso do protetor de vento. As maiores velocidades apresentaram tendência a um valor de nível de potência sonora elevado. Tosin et al. (2009) verificaram que as velocidades de deslocamento de $3,6 \mathrm{~km} \mathrm{~h}^{-1}$; $5,0 \mathrm{~km} \mathrm{~h}^{-1}$; e $6,8 \mathrm{~km} \mathrm{~h}^{-1}$ não afetaram o nível de potência sonora nas pistas de asfalto, concreto e terra batida.

$\mathrm{Na}$ análise de regressão polinomial para as pistas de ensaio, em função da velocidade de deslocamento, verificou-se que não há significância em nenhum grau da regressão. Entretanto, a regressão de terceiro grau explica $94,27 \%$ do fenômeno, para o equipamento que atende à norma IEC651, e 94,01\%, para o equipamento que atende à norma IEC 61672. Com base no apresentado, construiu-se o gráfico de representação dos modelos (Figura 5).

No gráfico (Figura 5) contendo os valores da potência sonora, em função da velocidade de deslocamento e das pistas de ensaio (terra batida e concreto), resultado da interação $(\mathrm{P} \times \mathrm{V})$, verificou-se que, à velocidade de $5 \mathrm{~km} \mathrm{~h}^{-1}$, o nível de potência sonora na pista de terra batida foi maior do que na de concreto, no entanto, à velocidade de $10,7 \mathrm{~km} \mathrm{~h}^{-1} \mathrm{e} 13,0 \mathrm{~km} \mathrm{~h}^{-1}$, ocorre o inverso, ou seja, o nível de potência sonora foi maior na pista de concreto. Neste caso, somente a marcha de maior velocidade $\left(13,0 \mathrm{~km} \mathrm{~h}^{-1}\right)$ proporcionou nível de potência sonora mais elevado na pista de concreto, corroborando os resultados encontrados por Vitória (2000).



Figura 5. Nível de potência sonora, em função da velocidade de deslocamento x pistas de ensaio (Petrolina, PE, 2009). 
Na regressão polinomial para a pista de ensaio de terra batida, utilizando-se os equipamento IEC651 e IEC61672, em função da velocidade de deslocamento (P [terra batida] x E x V), verificou-se que a regressão polinomial de terceiro grau foi significativa para os equipamentos, apresentando, também, o maior valor de $\mathrm{R}^{2}$ (Figura 6).

O gráfico (Figura 6) contendo as curvas de regressão para a pista de terra batida, com os equipamentos de medição em função da velocidade de deslocamento, apresentou, para o equipamento que atende à norma IEC61672, maior valor no nível de potência sonora, em todas as velocidades, estando acima do permitido pela NR-15 (MTE 2008), para uma jornada de trabalho de 8 horas, sem proteção auricular. Verificou-se que, à velocidade de $5 \mathrm{~km} \mathrm{~h}^{-1}$, há aumento no nível de potência sonora, nos equipamentos avaliados, sendo mais acentuado para o equipamento que atende à norma IEC61672. À velocidade de $5 \mathrm{~km} \mathrm{~h}^{-1}$, o trator utilizou a marcha L2 (segunda reduzida), que, devido ao acoplamento de engrenagens, pode ter produzido maior potência sonora. Souza et al. (2004) verificaram, para colhedoras de café, que as menores velocidades de deslocamento $\left(2,0-2,5 \mathrm{~km} \mathrm{~h}^{-1}\right)$ apresentaram maiores valores do nível de potência sonora.

$\mathrm{Na}$ regressão polinomial para a pista de concreto, com equipamento IEC651 e IEC61672, em função da velocidade de deslocamento, verificou-se que a regressão de primeiro grau foi significativa, no entanto, a equação de terceiro grau também foi significativa, devendo-se adotar o modelo de maior grau. Outro fator que ajuda na escolha do modelo é o $\mathrm{R}^{2}$, que foi maior para o terceiro grau.

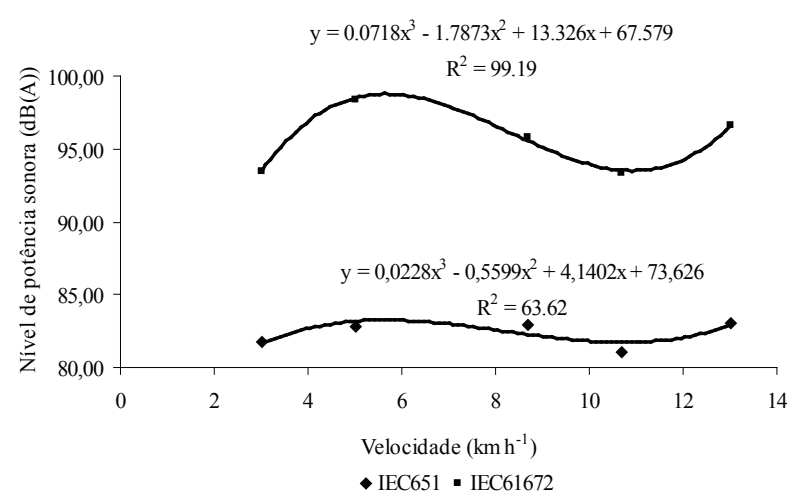

Figura 6. Nível de potência sonora na pista de terra batida, em função dos equipamentos de medição $\mathrm{x}$ velocidade de deslocamento (Petrolina, PE, 2009).
No gráfico (Figura 7) que contém as curvas de regressão da potência sonora na pista de concreto, em função dos equipamentos de medição e da velocidade de deslocamento, verificou-se que o equipamento que atende à norma IEC61672 apresentou maior valor de potência sonora do que o IEC651, sendo estes valores acima dos recomendados pela NR-15 (MTE 2008), para uma jornada de 8 horas. A potência sonora foi, praticamente, constante, ao longo das velocidades, com ligeiro aumento na marcha de maior velocidade $\left(13 \mathrm{~km} \mathrm{~h}^{-1}\right)$.

Ao se analisarem as duas pistas de ensaio (Figuras 6 e 7), verificaram-se valores semelhantes, em que o desvio padrão do equipamento IEC651 foi de $0,85 \mathrm{~dB}(\mathrm{~A})$ e de $1,10 \mathrm{~dB}(\mathrm{~A})$, para as pistas de terra batida e de concreto, respectivamente. Para o equipamento IEC61672, o desvio padrão foi de $2,30 \mathrm{~dB}(\mathrm{~A})$ e $2,00 \mathrm{~dB}(\mathrm{~A})$, respectivamente, para as pistas de terra batida e de concreto. Portanto, a avaliação nos dois tipos de pista não apresentaram diferenças significativas. Mialhe (1996) cita que, para as normas envolvendo ensaio de máquinas agrícolas, o teste deve ser realizado em pista de concreto. Tosin et al. (2009), ao avaliarem o nível de potência sonora em dois tratores $(55,2 \mathrm{~kW}$ e $80,9 \mathrm{~kW})$, em pistas de asfalto, concreto e terra batida, não verificaram influência no nível de potência sonora, e concluíram que os fatores que afetaram os ruídos foram a potência do motor e a presença da cabine.

O equipamento IEC61672 apresentou, em todas as condições, valores superiores de nível de potência sonora aos do IEC651. Especificamente para a norma IEC651, este equipamento é destinado

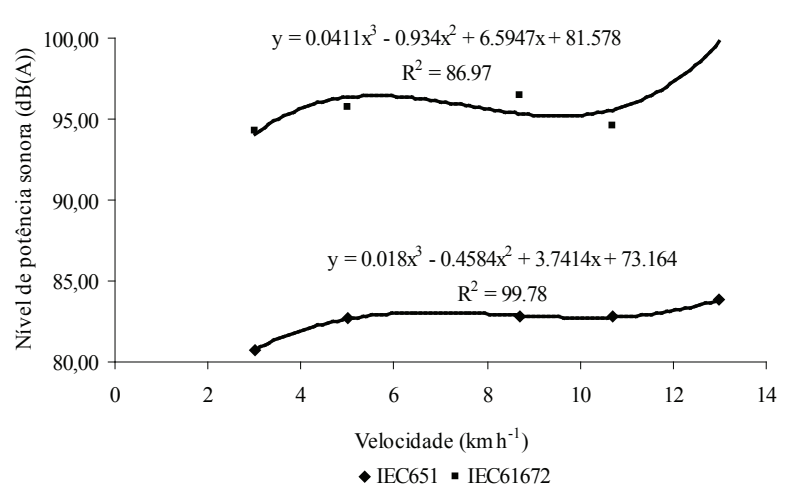

Figura 7. Nível de potência sonora na pista de concreto, em função dos equipamentos de medição $\mathrm{x}$ velocidade de deslocamento (Petrolina, PE, 2009). 
a uso em campo, no entanto, tal norma foi substituída pela IEC61672. Devido ao fato de o equipamento que atende à norma IEC651 estar com o protetor de vento, houve redução no nível de potência sonora medido por este equipamento.

Considerando-se as velocidades utilizadas para se medir o nível de potência sonora, em algumas condições, a marcha $2 \mathrm{~L}\left(5 \mathrm{~km} \mathrm{~h}^{-1}\right)$ apresentou valores maiores, no entanto, nas demais condições, a potência sonora foi maior na marcha de maior velocidade $\left(13,0 \mathrm{~km} \mathrm{~h}^{-1}\right)$.

\section{CONCLUSÕES}

1. Os níveis de ruído próximos ao operador, em condições de campo, foram superiores aos estabelecidos pelas normas, para uma exposição máxima de 8 horas diárias, sem o uso de protetor auricular.

2. À medida que se aumentou a rotação do motor do trator, houve incremento linear no nível de potência sonora, sendo que, a partir de 1.000 rpm, há necessidade do uso de proteção auricular.

3. A parte traseira do trator foi a que apresentou o menor valor para o nível de potência sonora, indicando que, a partir de quatro metros de distância, é possível trabalhar sem proteção auricular, em qualquer posição.

4. Os ensaios de níveis de potência sonora podem ser realizados em pista de terra batida e de concreto, sem diferenças significativas.

5. As velocidades de deslocamento afetaram o nível de potência sonora.

\section{AGRADECIMENTOS}

Ao Conselho Nacional de Desenvolvimento Científico e Tecnológico (CNPq), pela bolsa de iniciação científica concedida ao primeiro e segundo autores, e à Universidade Federal do Vale do São Francisco

(Univasf), especialmente à Seção de Apoio às Atividades de Campo.

\section{REFERENNCIAS}

ALVES, A. D. S. Nivel de potência sonora emitido por trator agrícola. 2009. 53 f. Trabalho de Conclusão de Curso (Graduação em Zootecnia)-Universidade Federal do Vale do São Francisco, Petrolina, 2009.
ASSOCIAÇÃO BRASILEIRA DE NORMAS TÉCNICAS (ABNT). NBR 10152: níveis de ruídos aceitáveis. Rio de Janeiro: ABNT, 1987.

ASSOCIAÇÃO BRASILEIRADENORMAS TÉCNICAS (ABNT). NBR NM-ISO 5353: máquinas rodoviárias, tratores e máquinas agrícolas e florestais: ponto de referência do assento. Rio de Janeiro: ABNT, 1999.

BAESSO, M. M. et al. Avaliação do nível de potência sonora emitido por um conjunto trator-pulverizador com e sem assistência de ar. Engenharia na Agricultura, Viçosa, v. 16, n. 4, p. 400-407, 2008.

BRASIL. Ministério da Agricultura. Levantamento exploratório: reconhecimento de solos do Estado de Pernambuco. Recife: Sudene, 1973. (Boletim técnico, 26).

CORTEZ, J. W. et al. Nível de pressão sonora em tratores agrícolas com e sem cabine. Cultivar Máquinas, Pelotas, n. 73, p. 28-30, 2008.

CUNHA, J. P. A. R.; TEODORO, R. E. F. Avaliação do nível de potência sonora em derriçadores e pulverizadores motorizados portáteis utilizados em lavouras de café. Bioscience Journal, Uberlândia, v. 22, n. 3, p. 71-77, 2006.

FERNANDES, J. C. Avaliação dos níveis de ruído em tratores e os problemas de audição em tratoristas. In: ENCONTRO NACIONAL DE ENGENHARIA DE PRODUÇÃO, 10., 2009, Belo Horizonte. Anais... Belo Horizonte: Enegep, 1990. 1 CD-ROM.

FERNANDES, J. C.; SANTOS, J. E. G.; ANDREATTA, J. A. Estudo do ruído em tratores com cabines. In: CONGRESSO BRASILEIRO DE ENGENHARIA AGRÍCOLA, 20., 1991, Londrina. Anais... Londrina: SBEA/Iapar, 1991. p. 1317-326.

MARTINELLI, M. M. Medição da pressão sonora em tratores agrícolas novos e suas adequações à N. R. 15 da C. L. T. In: FÓRUM AMBIENTAL DAALTA PAULISTA, 4., 2008, Tupã. Anais... Tupã: ANAP, 2008. 1 CD-ROM.

MIALHE, L. G. Máquinas agrícolas: ensaios e certificações. Piracicaba: Fealq, 1996.

MINISTÉRIO DO TRABALHO E DO EMPREGO (MTE). Atividades e operações insalubres: NR-15. 2008. Disponível em: <http://www.mtb.gov.br/legislacao/ normas_regulamentadoras/nr_15.asp $>$. Acesso em: 9 nov. 2008.

NORONHA, E. H.; TRAVAGLIA FILHO, U. J.; GARAVELLI, S. L. Quantificação dos níveis de ruídos num estande de tiros da PM do Distrito Federal. Humanitates, Brasília, DF, v. 1, n. 3, 2005. Disponível em: $<$ http://www.humanitates.ucb.br/3/ruido.htm $>$. Acesso em: 10 out. 2009. 
SILVEIRA, J. C. M. da et al. Níveis de ruído em função do raio de afastamento emitido por diferentes equipamentos em uma oficina agrícola. Engenharia na Agricultura, Viçosa, v. 15, n. 1, p. 66- 74, 2007.

SOUZA, L. H. et al. Níveis de ruído emitidos por uma recolhedora-trilhadora de feijão. Engenharia Agrícola, Jaboticabal, v. 24, n. 3, p. 745-749, 2004.

TOSIN, R. C.; LANÇAS, K. P.; ARAÚJO, J. A. Avaliação do ruído no posto de trabalho em dois tratores agrícolas. Energia na Agricultura, Botucatu, v. 24, n. 4, p. 108-118, 2009.
UNIVERSIDADE ESTADUAL PAULISTA (Unesp). Sistema para análises estatísticas: ESTAT. V. 2.0. Jaboticabal: Unesp, 1991.

VIGNA, G. P. et al. Nível de potência sonora e ergonomia no posto do operador em colhedoras. In: CONGRESSO BRASILEIRO DE ENGENHARIA AGRÍCOLA, 36., 2007, Bonito. Anais... Bonito: SBEA, 2007. 1 CD-ROM.

VITÓRIA, E. L. Avaliação do nível de ruído emitido por tratores em diferentes operações agrícolas. 2000. 76 f. Dissertação (Mestrado em Engenharia Agrícola)Universidade Federal de Viçosa, 2000. 